\title{
Patient medical costs for tuberculosis treatment and impact on adherence in China: a systematic review
}

Qian Long ${ }^{1,2^{*}}$, Helen Smith ${ }^{3}$, Tuohong Zhang ${ }^{4}$, Shenglan Tang ${ }^{3,5}$ and Paul Garner ${ }^{3}$

\begin{abstract}
Background: Charging for tuberculosis (TB) treatment could reduce completion rates, particularly in the poor. We identified and synthesised studies that measure costs of TB treatment, estimates of adherence and the potential impact of charging on treatment completion in China.
\end{abstract}

Methods: Inclusion criteria were primary research studies, including surveys and studies using qualitative methods, conducted in mainland China. We searched MEDLINE, PUBMED, EMBASE, Science Direct, HEED, CNKI to June 2010; and web pages of relevant Chinese and international organisations. Cost estimates were extracted, transformed, and expressed in absolute values and as a percentage of household income.

Results: Low income patients, defined at household or district level, pay a total of US\$ 149 to 724 (RMB 1241 to 5228) for medical costs for a treatment course; as a percentage of annual household income, estimates range from $42 \%$ to $119 \%$. One national survey showed $73 \%$ of TB patients at the time of the survey had interrupted or suspended treatment, and estimates from 9 smaller more recent studies showed that the proportion of patients at the time of the survey who had run out of drugs or were not taking them ranged from 3 to 25\%. Synthesis of surveys and qualitative research indicate that cost is the most cited reason for default.

Conclusions: Despite a policy of free drug treatment for TB in China, health services charge all income groups, and costs are high. Adherence measured in cross sectional surveys is often low, and the cumulative failure to adhere is likely to be much higher. These findings may be relevant to those concerned with the development and spread of multi-drug resistant TB. New strategies need to take this into account and ensure patient adherence.

\section{Background}

In China, over 130,000 people die from tuberculosis (TB) each year [1]. In 2004, 140,000 people were estimated to have multidrug resistant tuberculosis (MDR $\mathrm{TB})$, about one third of the total worldwide [2]. It is therefore particularly important that the health system ensures the delivery of full treatment to those with the disease.

China's health system was decentralised in the 1980's, and the central government budget for health facilities dropped to $10 \%$ of the total facilities' revenue by the early 1990s [3]. Health services were expected to generate revenue and manage surpluses [3]. Whilst

\footnotetext{
* Correspondence: qian.long@thl.fi

${ }^{1}$ School of Public Health, Chongqing Medical University, Chongqing, China Full list of author information is available at the end of the article
}

government controlled the price for basic health care, they also established a margin for drug sales so facilities could generate income and survive [4]. These initial reforms meant $\mathrm{TB}$ patients were charged like everyone else.

With increased TB incidence in the 1990's thought to be related to rural-urban migration [5], the government responded by abolishing fees for TB diagnosis and treatment. The TB control institutes are authorised to provide free TB drugs (6 months for new patients, 8 months if previously treated); and free X-ray examination at first visit, followed by a free sputum smear test. Accompanying this, health workers in general health facilities are obliged to refer TB suspects and patients to local TB dispensary for diagnosis confirmation and treatment. This national programme was organised centrally and conducted at county (district/city) level;
C Biomed Central

(C) 2011 Long et al; licensee BioMed Central Ltd. This is an Open Access article distributed under the terms of the Creative Commons Attribution License (http://creativecommons.org/licenses/by/2.0), which permits unrestricted use, distribution, and reproduction in any medium, provided the original work is properly cited. 
international agencies (e.g. World Bank) helped with subsidies, loans and technical advice [6]. Performance appeared remarkably high, with one report of a defaulter rate of $1.6 \%$ in 55,213 new patients [7].

Despite the central government commitment to free treatment, providers have found ways of generating revenue from TB patients. There is clear evidence, for example, that providers recommend treatment beyond the period of free drugs; request repeated investigation and follow up X-rays and blood tests; and prescribe liver protection and ancillary drugs $[1,8,9]$. All of these require payment, and are added on to the basic package of free care provided in the TB programme. Does this impact on adherence? Although the aggregate national statistics indicate very high completion rates, the programme is heavily target driven. It is possible that the official figures are perhaps optimistic estimates; they certainly hide regional and local variation where performance may be poorer. When carefully conducted, usually small, independent surveys are carried out that record a problem with adherence, there is a difficulty in interpretation: is this just a local problem, or more generalised? In such circumstances, a systematic review of the small studies can help examine this. Given the serious potential public health impact of incomplete TB treatment in 1,500,000 people under treatment each year, we sought to summarise reliable evidence relevant to these debates. We sought to answer three main questions:

1) How much do TB patients pay for medical care?

2) What is the range of estimates for default?

3) Is there any evidence of a link between medical care costs and adherence?

\section{Methods}

\section{Criteria for considering studies for this review}

This review included original research conducted in mainland China, with explicit methods so it was clear how the data had been collected. Criteria were specific to each question: for patient costs and completion rates, we sought surveys; for exploration of links between costs and adherence, we sought relevant surveys and qualitative data.

Inclusion criteria were carefully defined (Table 1) and applied. Studies where there was no information on the study design, study population and how data were collected were excluded. This excludes routine health information collected and collated by the health service. We included research in Chinese or English published between 1979 and June 2010.

\section{Search strategy}

We searched the following databases: MEDLINE, PUBMED, EMBASE, Science Direct, HEED with the following search terms: Tuberculosis, adherence, treatment, completion, cure; cost, financial burden; the poor, low income, vulnerable group, migrants; Directly Observed Treatment short-course (DOTs), initiatives, incentive, health insurance, New Cooperative Medical Scheme, China. The electronic search strategy for one database is in Table 2. One Chinese database (CNKI) was searched using terms "tuberculosis, treatment, cost, financial burden, adherence". This search was to identify studies in Chinese that were not included in English databases presented above. Google search and web pages of TB control of Chinese Ministry of Health and other international organisations (including the World Health Organization, World Bank) were screened in October 2010 and April 2011 for further relevant studies by searching on "(tuberculosis or TB control) and "China".

\section{Data extraction and transformation}

The first author identified studies based on examining titles, and then abstracts. Full texts of relevant abstracts were retrieved for further assessment. Data were extracted using a standard form. Uncertainties were resolved through discussion with co-authors and contact with study authors. Data were extracted onto a single form and the data extraction was checked by a second author. The main outcome we sought was medical cost

\section{Table 1 Inclusion criteria}

\begin{tabular}{|c|c|c|}
\hline Study question & Inclusion criteria & Data to be sought and extracted \\
\hline $\begin{array}{l}\text { 1. Medical costs } \\
\text { to TB patient }\end{array}$ & $\begin{array}{l}\text { Population-based surveys or facility-based surveys on TB } \\
\text { patients (including new and re-treatment patients) }\end{array}$ & $\begin{array}{l}\text { Total and average cost; cost as a proportion of annual } \\
\text { household income }\end{array}$ \\
\hline $\begin{array}{l}\text { 2. Adherence } \\
\text { rates }\end{array}$ & Population based surveys or cohort studies & Completion or cure per patient followed up \\
\hline \multirow[t]{2}{*}{$\begin{array}{l}\text { 3. Impact on } \\
\text { completion and } \\
\text { adherence }\end{array}$} & $\begin{array}{l}\text { Surveys must be population based, with household or individual } \\
\text { cost estimates from interviews and completion rates based on } \\
\text { verified health service data, or on patient reported length of } \\
\text { treatment }\end{array}$ & $\begin{array}{l}\text { A proportion of interrupted treatment because of financial } \\
\text { burden caused by TB treatment }\end{array}$ \\
\hline & $\begin{array}{l}\text { Qualitative studies that a) use recognised methods (in-depth } \\
\text { interviews, focus group discussions, or observation); and b) } \\
\text { describe the methods used in analysis (thematic analysis, } \\
\text { content analysis, grounded theory) }\end{array}$ & $\begin{array}{l}\text { Patient or provider views on cost and its influence on } \\
\text { treatment completion. Include evidence, in the form of } \\
\text { illustrative quotes or empirical data, for the statements }\end{array}$ \\
\hline
\end{tabular}


Table 2 Electronic search strategy for one database (Medline)

1 China [Mesh], ti, ab
2 Tuberculosis [Mesh], ti, ab
31 AND 2
4 Patient compliance [Mesh]
5 adherence OR DOT* OR (directly observed) ti, ab
6 (treatment completion) OR cost* OR finance* OR vulnerable OR
incentive* OR insurance OR fees OR monetary OR survey*) ti, ab
7 (low income $^{*}$ ) OR (vulnerable group*) OR migrant* ti, ab
84 OR 5 OR 6 OR 7
93 AND 8

of treatment; the variation in measuring cost of $\mathrm{TB}$ treatment required some data conversion, that we did using standard assumptions about length of treatment for six months. Furthermore, we extracted or calculated cost as a percentage of annual household income as an indicator to assess TB patient's financial burden by different study areas. We summarised data as brief narrative for outcome measure.

\section{Results}

\section{Description of studies}

Figure 1 presents the review profile. The search yielded 1143 studies for TB related diagnosis and treatment, of which 247 studies (including 12 studies having qualitative study design) potentially met the inclusion criteria. On the second screening, we excluded studies on the basis of the abstract. Sixtyeight studies were retrieved for full-text evaluation after screening the abstracts. Fifty-one studies were excluded because they reported routine health service surveillance data, or because they were duplicate

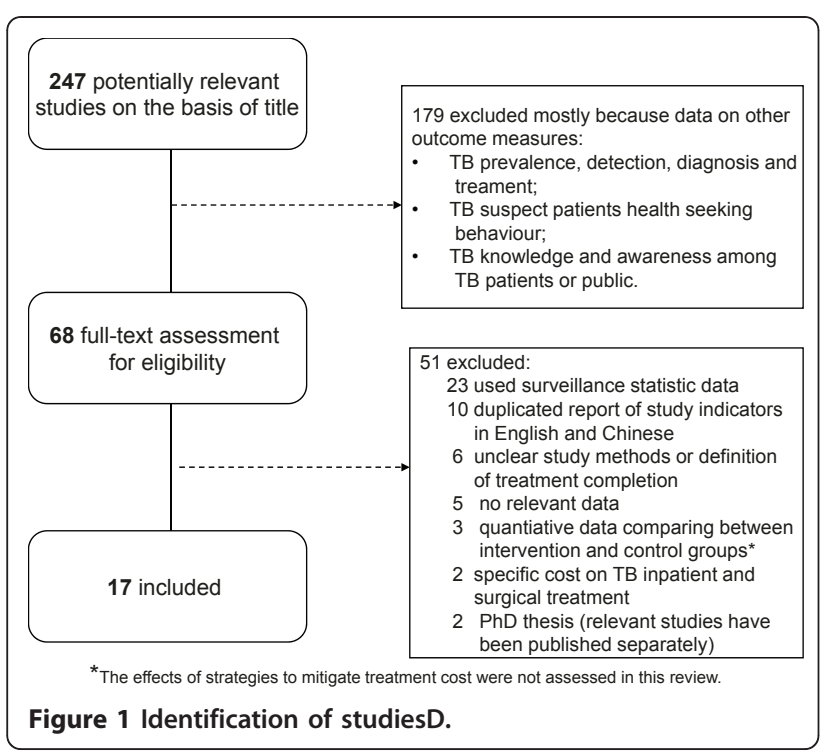

publications of a study already included, they provided no relevant data, or no study methods or the definition of treatment completion was not clear (Figure 1). Of the four national TB epidemic surveys between 1979 and 2000, only the 2000 study reported treatment completion rate and was included.

In all, 17 studies (14 in English and 3 in Chinese) met the inclusion criteria for questions 1-3. The study design included surveys (14), both quantitative and qualitative methods (2), and qualitative design only (1). Studies were national (2); limited to 4 selected provinces in all regions (1); eastern (7); western (4); and central (2); and unknown region due to authors concerns about confidentiality (1). Most counties studied were middle or low income; eleven studies were concerned wholly with counties designated as World Bank/TB Programme areas where TB drugs were supplied for free (Table 3).

\section{Medical costs}

Nine studies provided estimates of total medical costs per treatment (excluding transport and food) (Liu 2010, Meng 2004, Jackson 2006, Zhang 2007, Xu 2010, Liu 2007, Xu 2006, Zhan 2004 and Huang 2009) (Table 4):

- RMB 1584 (US\$ 220) per household for one treatment was the average medical cost per treatment among low income groups in 8 counties surveyed in a study $(\mathrm{n}=24)$, representing $93 \%$ of the total annual household income in this group [8].

- RMB 2241 to 3070 (US\$ 270 to 371) per household for one treatment was the range for the average cost in each of 2 poor counties surveyed in a study $(n=146)$, representing $57 \%$ to the maximum of $119 \%$ of average annual household income [10].

- RMB 1940 (US\$242) was the total average out-ofpocket payment for medical costs from diagnosis to completion representing 39\% of annual household income in one carefully conducted study. The authors interviewed the same patients $(\mathrm{n}=144)$ on two occasions in 4 counties where drugs were supplied for free [11].

- RMB 1241 (US\$ 149) of annual household medical expense among low income households with a TB patient (as a proxy indicator to estimate medical cost for TB treatment) was the finding in the fourth study $(\mathrm{n}=80)$, representing $42 \%$ of annual household income in this group [12].

- RMB 4079 to 5228 (US\$ 565 to 724 ) was the range for annual household medical expense in two poor counties (as a proxy indicator to reflect financial situation of TB patients) in a fifth study $(\mathrm{n}=156)$, representing $65 \%$ to $84 \%$ of average annual household income [13]. 
Table 3 Description of included studies

\begin{tabular}{|c|c|c|c|c|c|c|c|c|c|c|c|}
\hline \multirow{2}{*}{$\begin{array}{l}\text { Author } \\
\text { year }\end{array}$} & \multirow{2}{*}{$\begin{array}{l}\text { Data } \\
\text { collected }\end{array}$} & \multirow{2}{*}{$\begin{array}{l}\text { TB } \\
\text { patient } \\
\text { (n) }\end{array}$} & \multirow[t]{2}{*}{ Province } & \multirow{2}{*}{$\begin{array}{l}\text { Sample } \\
\text { unit }\end{array}$} & \multirow{2}{*}{$\begin{array}{l}\text { Income } \\
\text { category, } \\
\text { status of } \\
\text { free } \\
\text { treatment }\end{array}$} & \multirow[t]{2}{*}{ Methods } & \multirow[t]{2}{*}{ Outcomes } & \multirow{2}{*}{$\begin{array}{l}\text { Data } \\
\text { collection }\end{array}$} & \multicolumn{3}{|c|}{$\begin{array}{l}\text { Questions } \\
\mathrm{a}\end{array}$} \\
\hline & & & & & & & & & 1 & 2 & 3 \\
\hline $\begin{array}{l}\mathrm{Liu} \\
2010^{8}\end{array}$ & 2007 & 163 & $\begin{array}{l}\text { Chongqing } \\
\text { Sichuan }\end{array}$ & $\begin{array}{l}4 \\
\text { counties } \\
\text { in each } \\
\text { province }\end{array}$ & $\begin{array}{l}8 \text { mixed } \\
\text { income, } \\
\text { All free }\end{array}$ & Patient survey & $\begin{array}{l}\text { Reported cost in previous } \\
\text { month on TB drugs and } \\
\text { tests }\end{array}$ & $\begin{array}{l}\text { Postgraduate } \\
\text { students }\end{array}$ & $\bullet$ & & \\
\hline $\begin{array}{l}\text { Zhan } \\
2004^{9}\end{array}$ & 2000-2001 & 257 & $\begin{array}{l}\text { Shandong } \\
\text { Jiangsu }\end{array}$ & Counties & $\begin{array}{l}3 \text { middle, } \\
1 \text { free } 2 \\
\text { not free }\end{array}$ & Patient survey & $\begin{array}{l}\text { Reported total medical cost } \\
\text { per treatment }\end{array}$ & Health staff & $\bullet$ & & \\
\hline $\begin{array}{l}\text { Meng } \\
2004^{10}\end{array}$ & 2000 & 312 & Shandong & Counties & $\begin{array}{l}2 \text { middle } \\
\text { income, } \\
2 \text { poor: All } \\
\text { free }\end{array}$ & Patient survey & $\begin{array}{l}\text { Reported total medical } \\
\text { annual cost per TB patient }\end{array}$ & $\begin{array}{l}\text { Medical } \\
\text { students and } \\
\text { health staff }\end{array}$ & $\bullet$ & & \\
\hline $\begin{array}{l}\mathrm{Liu} \\
2007^{14}\end{array}$ & 2004 & 889 & $\begin{array}{l}\text { Fujian } \\
\text { Henan } \\
\text { Liaoning } \\
\text { Xinjiang }\end{array}$ & $\begin{array}{l}3 \\
\text { counties } \\
\text { in each } \\
\text { province }\end{array}$ & $\begin{array}{l}12 \text { poor, } \\
\text { All free }\end{array}$ & Patient survey & $\begin{array}{l}\text { Reported direct household } \\
\text { cost per treatment }\end{array}$ & $\begin{array}{l}\text { Health staff } \\
\text { from non-TB } \\
\text { system and } \\
\text { university } \\
\text { students }\end{array}$ & $\bullet$ & & \\
\hline $\begin{array}{l}\text { Huang } \\
2009^{16}\end{array}$ & 2007 & 100 & Hunan & Counties & $\begin{array}{l}10 \\
\text { unknown, } \\
\text { All free }\end{array}$ & Patient survey & $\begin{array}{l}\text { Reported total medical cost } \\
\text { per treatment }\end{array}$ & Unknown & $\bullet$ & & \\
\hline $\begin{array}{l}\mathrm{Xu} \\
2006^{15}\end{array}$ & $2002-2003$ & 465 & Jiangsu & Counties & $\begin{array}{l}2 \text { middle } \\
\text { income, } \\
1 \text { free } 1 \\
\text { not free }\end{array}$ & $\begin{array}{l}\text { Longitudinal: } 4 \\
\text { interviews per } \\
\text { patient }{ }^{\text {b }}\end{array}$ & $\begin{array}{l}\text { 1. Reported total medical } \\
\text { cost for treatment } 2 \text {. Non- } \\
\text { adherence rate }\end{array}$ & TB specialist & $\bullet$ & $\bullet$ & \\
\hline $\begin{array}{l}\mathrm{Xu} \\
2010^{13}\end{array}$ & 2008 & 501 & Shandong & Counties & $\begin{array}{l}6 \text { mixed } \\
\text { income, } \\
\text { All free }\end{array}$ & Patient survey & $\begin{array}{l}\text { 1. Reported annual } \\
\text { household medical cost in } \\
\text { household with TB patient } \\
\text { 2. Non-adherence rate }\end{array}$ & $\begin{array}{l}\text { Independent } \\
\text { investigator } \\
\text { with help of } \\
\text { TB staff }\end{array}$ & $\bullet$ & $\bullet$ & \\
\hline $\begin{array}{l}\text { Zhang } \\
2007^{12}\end{array}$ & 2002 & 182 & $\begin{array}{l}\text { Whole of } \\
\text { rural China }\end{array}$ & $\mathrm{N}-\mathrm{A}$ & $\mathrm{N}-\mathrm{A}$ & Patient survey & $\begin{array}{l}\text { 1. Reported annual } \\
\text { household medical cost in } \\
\text { household with TB patient } \\
\text { 2. Influence of charging on } \\
\text { treatment }\end{array}$ & $\begin{array}{l}\text { Township } \\
\text { health } \\
\text { workers }\end{array}$ & $\bullet$ & & $\bullet$ \\
\hline $\begin{array}{l}\text { Jackson } \\
2006^{11}\end{array}$ & $2002-2005$ & 160 & Henan & Counties & $\begin{array}{l}4 \text { mixed } \\
\text { income, } \\
\text { All free }\end{array}$ & $\begin{array}{l}\text { Longitudinal: } 2 \\
\text { interviews per } \\
\text { patient }^{c}\end{array}$ & $\begin{array}{l}\text { 1. Reported total medical } \\
\text { cost per completed } \\
\text { treatment } 2 \text {. Non- } \\
\text { adherence rate } 3 \text {. Influence } \\
\text { of charging on treatment }\end{array}$ & TB specialist & $\bullet$ & $\bullet$ & $\bullet$ \\
\hline $\begin{array}{l}\text { Wang } \\
2007^{18}\end{array}$ & 2006 & 130 & Yunnan & Counties & $\begin{array}{l}10 \text { mixed } \\
\text { income, } \\
\text { All free }\end{array}$ & Patient survey & Non-adherence rate & $\begin{array}{l}\text { Village } \\
\text { doctors and } \\
\text { local CDC } \\
\text { staff }\end{array}$ & & $\bullet$ & \\
\hline $\begin{array}{l}\text { Wang } \\
2009^{21}\end{array}$ & 2004-2007 & 607 & unknown & Counties & $\begin{array}{l}2 \text { high } \\
\text { income, } \\
\text { All free }\end{array}$ & Patient survey & Non-adherence rate & $\begin{array}{l}\text { Independent } \\
\text { investigator }\end{array}$ & & $\bullet$ & \\
\hline $\begin{array}{l}\text { NESS-TB } \\
2002^{17 d}\end{array}$ & 2000 & $1278^{e}$ & $\begin{array}{l}\text { Whole of } \\
\text { China }\end{array}$ & $\mathrm{N}-\mathrm{A}$ & $\mathrm{N}-\mathrm{A}$ & Patient survey & $\begin{array}{l}\text { 1. Non-adherence rate } 2 . \\
\text { Influence of charging on } \\
\text { adherence }\end{array}$ & unknown & & $\bullet$ & $\bullet$ \\
\hline $\begin{array}{l}\mathrm{Hu} \\
2008^{19}\end{array}$ & 2004 & 405 & Chongqing & Counties & $\begin{array}{l}4 \text { mixed } \\
\text { income, } \\
2 \text { free } 2 \\
\text { not free }\end{array}$ & $\begin{array}{l}\text { 1. Patient survey } 2 . \\
\text { In-depth interview }\end{array}$ & $\begin{array}{l}\text { 1. Non-adherence rate } 2 . \\
\text { Influence of charging on } \\
\text { adherence }\end{array}$ & $\begin{array}{l}\text { Postgraduate } \\
\text { students }\end{array}$ & & $\bullet$ & $\bullet$ \\
\hline $\begin{array}{l}\mathrm{Xu} \\
2009^{20}\end{array}$ & 2006 & 670 & Jiangsu & Counties & $\begin{array}{l}13 \text { mixed } \\
\text { income, } \\
\text { All free }\end{array}$ & $\begin{array}{l}\text { 1. Patient survey } 2 \text {. } \\
\text { In-depth interview }\end{array}$ & $\begin{array}{l}\text { 1. Non-adherence rate } 2 . \\
\text { Influence of charging on } \\
\text { adherence }\end{array}$ & TB staff & & $\bullet$ & $\bullet$ \\
\hline $\begin{array}{l}\mathrm{Ai} \\
2010^{22}\end{array}$ & 2006-2007 & 659 & Shaanxi & Counties & $\begin{array}{l}30 \text { poor, } \\
\text { All free }\end{array}$ & Patient survey & $\begin{array}{l}\text { 1. Non-adherence rate } 2 \text {. } \\
\text { Influence of charging on } \\
\text { adherence }\end{array}$ & Unknown & & $\bullet$ & $\bullet$ \\
\hline
\end{tabular}


Table 3 Description of included studies (Continued)

\begin{tabular}{|c|c|c|c|c|c|c|c|c|c|c|}
\hline $\begin{array}{l}\text { Sun } \\
2007^{23}\end{array}$ & 2004-2005 & 473 & Jiangsu & County & $\begin{array}{l}1 \\
\text { unknown, } \\
\text { Free }\end{array}$ & $\begin{array}{l}\text { Longitudinal: } 3 \\
\text { interviews at } 2^{\text {nd }} \text {, } \\
5^{\text {th }} \text { treatment } \\
\text { month and } \\
\text { treatment } \\
\text { completion }\end{array}$ & $\begin{array}{l}\text { 1. Non-adherence rate } 2 . \\
\text { Influence of charging on } \\
\text { adherence }\end{array}$ & TB staff & $\bullet$ & $\bullet$ \\
\hline $\begin{array}{l}\mathrm{Xu} \\
2004^{24}\end{array}$ & unknown & 30 & Jiangsu & Counties & $\begin{array}{l}2 \text { middle } \\
\text { income, } \\
1 \text { free } 1 \\
\text { not free }\end{array}$ & $\begin{array}{l}\text { Focus Group } \\
\text { Discussions (FGDs) }\end{array}$ & $\begin{array}{l}\text { Influence of charging on } \\
\text { adherence }\end{array}$ & $\begin{array}{l}\text { Researcher } \\
\text { and } \\
\text { postgraduate } \\
\text { students }\end{array}$ & & $\bullet$ \\
\hline
\end{tabular}

$\mathrm{N}-\mathrm{A}$ : not applicable

a In the column "Questions", point refer to which question the paper contributes data to

${ }^{b}$ Good follow up (>92\% complete all interviews);

c After 10-12 months 11 people died and five could not be located.

${ }^{d}$ NESS-TB: National technical steering group of the epidemiological sampling survey for tuberculosis

e 900 new cases and 378 patients who received treatment

Table 4 Annual medical costs for one TB treatment (RMB). Excludes costs for diagnosis

\begin{tabular}{|c|c|c|c|c|c|c|c|}
\hline Study & Methods & $n$ & Variables & $\begin{array}{l}\text { County or patient } \\
\text { group }\end{array}$ & $\begin{array}{c}\text { TB } \\
\text { treatment }\end{array}$ & $\begin{array}{l}\text { Annual } \\
\text { cost }\end{array}$ & $\begin{array}{l}\text { \% of annual } \\
\text { household } \\
\text { income }\end{array}$ \\
\hline $\begin{array}{c}\text { Liu } \\
2010^{8}\end{array}$ & $\begin{array}{c}\text { Method: Survey Includes: All TB patients } \\
\text { Extent: } 8 \text { counties }\end{array}$ & 163 & $\begin{array}{l}\text { Average expenditure in } \\
\text { previous month on TB } \\
\text { drugs and tests }\end{array}$ & $\begin{array}{l}\text { Income below } \\
\text { poverty line } \\
<3720 / \text { year }(n= \\
24)\end{array}$ & Free & $1584^{a}$ & $93 \%$ \\
\hline $\begin{array}{l}\text { Meng } \\
2004^{10}\end{array}$ & $\begin{array}{l}\text { Method: Survey Includes: All patients Extent: } \\
\qquad 4 \text { counties }\end{array}$ & 312 & $\begin{array}{l}\text { Average total medical } \\
\text { annual cost per patient }\end{array}$ & $\begin{array}{l}\text { Poor county } 1 \text { ( } n= \\
81) \\
\text { Poor county } 2 \text { ( } n= \\
65)\end{array}$ & $\begin{array}{l}\text { Free } \\
\text { Free }\end{array}$ & $\begin{array}{l}3070 \\
2241\end{array}$ & $\begin{array}{l}119 \% \\
57 \%\end{array}$ \\
\hline $\begin{array}{l}\text { Jackson } \\
2006^{11}\end{array}$ & $\begin{array}{l}\text { Method: Case control Costs prospective } \\
\text { Includes: All patients Extent: } 4 \text { counties }\end{array}$ & 160 & $\begin{array}{l}\text { Direct medical costs per } \\
\text { completed treatment }\end{array}$ & $\begin{array}{l}\text { Mixed income }(n= \\
144)\end{array}$ & Free & 1940 & $39 \%$ b \\
\hline $\begin{array}{l}\text { Zhang } \\
2007^{12}\end{array}$ & $\begin{array}{c}\text { Method: Household survey Number: } \\
\text { 143,991 people Includes: TB patients Extent: } \\
\text { National }\end{array}$ & 180 & $\begin{array}{l}\text { Annual household medical } \\
\text { cost }\end{array}$ & $\begin{array}{l}\text { Low income }(n= \\
80)\end{array}$ & Mixed & 1241 & $42 \%$ \\
\hline $\begin{array}{c}\mathrm{Xu} \\
2010^{13}\end{array}$ & $\begin{array}{c}\text { Method: Survey Includes: All TB patients } \\
\text { Extent: } 6 \text { counties }\end{array}$ & 501 & $\begin{array}{l}\text { Annual household medical } \\
\text { cost }\end{array}$ & $\begin{array}{l}\text { Poor county } 1 \text { ( } n= \\
68) \\
\text { Poor county } 2(n= \\
88)\end{array}$ & $\begin{array}{l}\text { Free } \\
\text { Free }\end{array}$ & $\begin{array}{l}5228 \\
4079\end{array}$ & $\begin{array}{l}65 \% \\
84 \%\end{array}$ \\
\hline$\underset{2007^{14}}{\operatorname{Liu}}$ & $\begin{array}{c}\text { Method: Survey Includes: Random sample } \\
\text { (TB patients) Extent: } 3 \text { poor counties in } \\
\text { each of } 4 \text { provinces }\end{array}$ & 889 & $\begin{array}{l}\text { Direct medical cost per } \\
\text { treatment }\end{array}$ & $\begin{array}{l}\text { Province } 1(n= \\
217)) \\
\text { Province } 2(n= \\
228) \\
\text { Province } 3(n= \\
223) \\
\text { Province } 4(n= \\
221)\end{array}$ & $\begin{array}{l}\text { Free } \\
\text { Free } \\
\text { Free } \\
\text { Free }\end{array}$ & $\begin{array}{c}775 \\
800 \\
1760 \\
445\end{array}$ & $\begin{array}{l}8 \% c \\
11 \% \\
27 \% \\
5 \%\end{array}$ \\
\hline $\begin{array}{c}X u \\
2006^{15}\end{array}$ & $\begin{array}{l}\text { Method: Cohort study Includes: All TB } \\
\text { patients Extent: } 2 \text { counties }\end{array}$ & 465 & $\begin{array}{l}\text { Direct medical cost per } \\
\text { treatment }\end{array}$ & $\begin{array}{l}\text { Middle county } 1 \text { ( } \mathrm{n} \\
=183 \text { ) } \\
\text { Middle county } 2 \text { ( } \mathrm{n} \\
=282 \text { ) }\end{array}$ & $\begin{array}{c}\text { Free } \\
\text { Charged }\end{array}$ & $\begin{array}{c}90 \\
704\end{array}$ & $\begin{array}{l}2 \%{ }^{d} \\
12 \%\end{array}$ \\
\hline $\begin{array}{l}\text { Zhan } \\
2004^{9 e}\end{array}$ & $\begin{array}{l}\text { Method: Survey Includes: All patients } \\
\text { Extent: } 3 \text { counties }\end{array}$ & 257 & $\begin{array}{l}\text { Direct medical costs per } \\
\text { treatment }\end{array}$ & $\begin{array}{l}\text { Middle county } 1 \text { ( } \\
=46 \text { ) } \\
\text { Middle county } 2 \text { ( } \mathrm{n} \\
=105 \text { ) }\end{array}$ & $\begin{array}{c}\text { Free } \\
\text { Charged }\end{array}$ & $\begin{array}{l}1517 \\
1754\end{array}$ & NA \\
\hline $\begin{array}{l}\text { Huang } \\
2009^{16}\end{array}$ & $\begin{array}{l}\text { Method: Survey Includes: Sample (TB } \\
\text { patients) Extent: } 10 \text { counties }\end{array}$ & 100 & $\begin{array}{l}\text { Direct medical costs per } \\
\text { treatment }\end{array}$ & Unknown & Free & 1635 & NA \\
\hline
\end{tabular}

NA: not available

${ }^{a}$ Monthly costs estimated multiplied by six; Monthly household income reported multiplied by twelve

${ }^{b}$ From reported average annual household income of the TB patients interviewed

${ }^{c}$ From total cost as a percentage of annual household expenditure

d Based on reported average annual household income of the TB patients interviewed

e Cost in US\$ converted to RMB by exchange rate used in the study. One county excluded as data from health worker estimates

${ }^{f}$ Random sample in one of the three counties 
- RMB 445 to 1760 (US\$ 54 to214) was the range for the average medical cost per treatment in each of 3 poor counties of 4 provinces in a sixth study, representing $5 \%$ to $27 \%$ of annual household expenditure [14].

- RMB 90 to 1754 (US\$10 to 213) was the direct medical cost per treatment by county with or without free treatment in two studies $[9,15]$.

- RMB 1635 (US\$ 199) was the average direct medical cost from diagnosis to treatment completion in 10 counties with free treatment in one study [16].

Relationships with cost: In 3 studies (Liu 2010, Meng 2004 and Zhang 2007) $[8,10,12]$ charges were disaggregated by household income, there was no obvious relationship between charges and the income category of the household; and in Meng, between poor and middle income counties (Table 5). However, the average cost as a proportion of total household income increased in the low income groups.

In two studies (Zhan 2004, Xu 2006) [9,15] comparing counties, the "free treatment" counties had diagnostic costs 2-3 times that the other counties that charge for TB drugs, suggesting transfer of costs from one function of the TB service to another. In one of the two studies (Zhan 2004), drug treatment costs were high despite this being a county with free treatment.

All but one study gave a single figure for "medical costs". The exception was Liu (2010) [8]: the authors asked patients about the cost components, and showed these consisted of prescribing of liver protection, immune stimulant and anti-inflammatory drugs, and monthly X-ray and blood tests, with an average of RMB 287 (US\$ 40) charged to each patient in the previous month. In the low income group, cost of liver protection drugs was $71 \%$ of patient monthly income; $32 \%$ and $63 \%$ of patient income being spent on ancillary drugs and tests respectively.

\section{Adherence to treatment}

Ten studies were surveys in which the investigators asked whether people who should have been on treatment were actually taking their drugs (Table 6). The large national sample survey in 2000 showed that $73 \%$ of patients underwent interrupted or suspended treatment (39\% in TB services system and $79 \%$ in general health system) [17].

In 6 smaller studies (Xu 2010, Wang 2007, Hu 2008, Xu 2009, Wang 2009 and Ai 2010), failure to follow standard treatment course varied from $3 \%$ to $25 \%$ across these studies [13,18-22]. Although each study used different outcomes, all appeared to measure the point prevalence of non-adherence; this does not provide the cumulative effect over the entire treatment period. Three studies followed up a cohort (Jackson 2006, Xu 2006 and Sun 2007) reported rate of incomplete treatment course from $5 \%$ to $13 \%[11,15,23]$.

\section{Association between patient costs and adherence}

The national survey and 4 smaller studies examined reasons why patients had interrupted treatment (National TB epidemiological survey 2002, Jackson 2006, Zhang 2007, Xu 2009 and Ai 2010). The percentage of nonadherence for reasons of cost varied from $3 \%$ to $45 \%$ of the total number of patients (Table 7) [11,12,17,20,22]. One study (Sun, 2007) examined the association between treatment cost and adherence showing higher cost with higher likelihood of non-adherence [23].

In the three studies that met our inclusion criteria for qualitative designs, a link between costs and adherence

Table 5 Estimates of charging: medical cost for TB care by income category (RMB)

\begin{tabular}{|c|c|c|c|c|c|c|}
\hline \multirow[t]{2}{*}{ Study } & \multirow[t]{2}{*}{ Cost variable } & \multirow[t]{2}{*}{ County } & \multicolumn{3}{|c|}{ Income category $^{a}$} & \multirow{2}{*}{$\begin{array}{l}\text { Average cost, \% annual } \\
\text { household income }\end{array}$} \\
\hline & & & Low & Middle & High & \\
\hline \multirow{4}{*}{$\begin{array}{l}\text { Meng } \\
2004^{10}\end{array}$} & Total medical cost & Middle & 2099 & 2826 & 3105 & $75 \%$ \\
\hline & & Middle & 2104 & 1449 & 1680 & $27 \%$ \\
\hline & & Poor & 4015 & 2011 & 3238 & $119 \%$ \\
\hline & & Poor & 1843 & 2054 & 2894 & $57 \%$ \\
\hline $\begin{array}{l}\text { Zhang } \\
2007^{12}\end{array}$ & $\begin{array}{l}\text { Annual household medical cost in household } \\
\text { with TB patient }\end{array}$ & $\begin{array}{l}\text { Whole rural } \\
\text { China }\end{array}$ & $\begin{array}{l}1241 \\
(42 \%)^{b}\end{array}$ & $\begin{array}{l}2061 \\
(28 \%)^{b}\end{array}$ & $\begin{array}{l}2090 \\
(19 \%)^{b}\end{array}$ & NA \\
\hline Liu $2010^{8}$ & Monthly medical cost & Mixed & $\begin{array}{l}264 \\
(185 \%)^{c}\end{array}$ & $\begin{array}{l}280 \\
(22 \%)^{c}\end{array}$ & $332(8 \%)^{c}$ & $18 \%^{c}$ \\
\hline
\end{tabular}

NA: not available

a In Meng's study, Income category was classified by per capita income per year (Low $\leq 500$; Middle 500-1000; High > 1000)

In Zhang's study, each income category contained a third of the total number of households;

In Liu's study, income category was classified by family monthly income (Low < 310; Middle 310-2600; High > 2600);

${ }^{b}$ Average annual household medical cost in household with TB patient as a percentage of annual household income in each income group;

${ }^{c}$ Average monthly medical cost as a percentage of family monthly income in each income group and at an average level. 
Table 6 Non-adherence estimates from studies

\begin{tabular}{|c|c|c|c|c|}
\hline Study & Study design & $\mathrm{n}$ & Definition of non-adherence & $\begin{array}{l}\text { Non-adherence } \\
\text { rate }\end{array}$ \\
\hline $\mathrm{Xu} 2006^{15}$ & $\begin{array}{l}\text { Cohort: } 4 \text { interviews per } \\
\text { patient }\end{array}$ & 465 & Did not complete treatment course & $5 \%-9 \%$ \\
\hline Jackson $2006^{11}$ & $\begin{array}{l}\text { Cohort: } 2 \text { interviews per } \\
\text { patient }\end{array}$ & 144 & Partial treatment & $11 \%$ \\
\hline Sun $2007^{23}$ & $\begin{array}{l}\text { Cohort: } 3 \text { interviews per } \\
\text { patient }\end{array}$ & 473 & Interrupted or defaulted treatment & $13 \%$ \\
\hline $\mathrm{Xu} 2010^{13}$ & Patient survey & 501 & Defaulted and failed treatment & $25 \%$ \\
\hline Wang $2007^{18}$ & Patient survey & 130 & $\begin{array}{l}\text { Stopped treatment }>2 \text { weeks or missed over } 20 \% \\
\text { of pack }\end{array}$ & $3 \%$ or $4 \%$ \\
\hline $\mathrm{Hu} 2008^{19}$ & Patient survey & 401 & Missing last three doses, or no stock $>1$ week & $13 \%$ \\
\hline $\mathrm{Xu} 2009^{20}$ & Patient survey & 670 & Missing $10 \%$ or more of the total pack & $12 \%$ \\
\hline Wang $2009^{21}$ & Patient survey & 537 & Did not complete treatment course & $15 \%$ \\
\hline Ai $2010^{22}$ & Patient survey & 659 & Defaulted treatment ${ }^{a}$ & $12 \%$ \\
\hline $\begin{array}{l}\text { National TB epidemiological survey } \\
2002^{17}\end{array}$ & Patient survey & 378 & Interrupted or suspended treatment & $73 \%$ \\
\hline
\end{tabular}

a 82 defaulted from treatment for which 20 died.

was identified in them all. Xu (2004), conducted in a county that did not implement free treatment, reported that treatment interruption occurred frequently due to shortage of money, particularly after the first two months of treatment [24]. Under free treatment, Xu (2009) indicated that patients complained of adverse reactions and low quality of free drugs; and doctors admitted to recommending patients buy drugs rather than taking the free drugs. Both patients and local doctors reported that privately purchased drugs imposed a higher financial burden to TB patients, sometimes resulting in interrupted treatment [20]. In another study (Hu 2008) the high cost of treatment was one of the main reasons mentioned by patients who interrupted treatment and village doctors agreed that financial barriers led to default [19]. In two studies, patients specifically described having to pay extra for examinations and additional drugs (mainly liver protection drugs) related to treatment. Patients reported that these additional costs were significant, accounted for a large part of family income, and were related to treatment adherence $[19,20]$.

\section{Discussion}

\section{Summary of findings}

Overall, low income patients, defined at household or district level, pay large amounts of money for medical treatment of TB, ranging from US\$ 149 to 724 (RMB 1241 to 5228); as a percentage of annual household income, estimates range from $42 \%$ to $119 \%$. One national survey showed $73 \%$ of TB patients at survey had interrupted or suspended treatment, and estimates from 9 smaller more recent studies of showed that the proportion of patients at the time of the survey who had run out of drugs or were not taking them ranged from 3 to $25 \%$. Synthesis of surveys and qualitative research indicate that cost is the most cited reason for default.

\section{Methodological and other potential limitations Limitations of primary studies}

Medical costs of TB treatment were measured in different ways in each study. This includes two studies which used annual household medical expenditure as proxy indicator. Obtaining data on expenditure is complex. In these studies, there is likely to be considerable variation in estimates due

Table 7 Studies reporting relationship between charging and adherence

\begin{tabular}{lll}
\hline $\begin{array}{l}\text { Study } \\
\text { design }\end{array}$ & Study & Comment \\
\hline Survey & $\begin{array}{l}\text { National TB epidemiological } \\
\text { survey } 2002^{17} \\
\text { Zhang } 2007^{12}\end{array}$ & $45 \%(121 / 272)$ of patients having interrupted or suspended treatment due to financial difficulty \\
& Xu $2009^{20}$ & $9 \%$ of households with TB suspects (2308) or patients defaulted due to financial burden \\
& Ai $2010^{22}$ & $\begin{array}{l}16 \%(13 / 82) \text { of non-adherence patients interrupted treatment because of high medical costs of the } \\
\text { treatment } \\
\text { Cackson } 2006^{11}\end{array}$ \\
& Sun of patients who interrupted treatment (43) because of financial difficulty \\
& $3 \%(5 / 159)$ was too poor to begin treatment \\
& & Patients who were charged high medical cost were more likely to interrupt treatment than patients \\
& having lower medical costs
\end{tabular}


to a) the rigor of the questions; b) the parameters of cost estimated; and c) the region, province and locality where the research was carried out, as costs vary considerably in China. Few studies provided disaggregated data of the different components of the additional cost (such as tests, additional drugs, or extending treatment).

\section{Limitations of our review}

This study reviews population-based studies other than routine register data considering reliability of information, but unpublished studies are not included due to practical constraints. As well as the limitations in the evidence we reviewed, we made assumptions in trying to obtain a uniform measure of cost across studies, which could contribute additional uncertainty to the estimates. Another limitation of our study is that the conclusions are based on indirect evidence and inference of an effect from very varied data from different regions of China: we cannot be clear how far all these findings are generalizable across the country.

\section{Interpretation}

In a primary health care system where fee for service is normal, the Chinese government commendably made TB drugs free. However, charges remain universal. It appears free drugs are overlaid on an existing health system based on fee for service, which proves problematic given the existing organisational norms and culture. The charges arise from additional and often unnecessary drugs and tests beyond those supplied through the national programme [8].Thus the revenue-driven practices in the general health system are influencing $\mathrm{TB}$ control, including overprovision, poor referral (to keep patients), and high hospitalisation costs [1,9].

We did identify policies to try and mitigate treatment cost but data were limited. A number of pilot schemes are underway, including decentralisation of TB diagnosis and case management services to the township level [25]; travel subsidies to get to clinic; payment to doctors for directly observing treatment [26]; free treatment for migrant patients [27]; and schemes linked to the New Cooperative Medical Scheme (NCMS), including case based payment.

The NCMS in China since 2003 has reached over 90\% of the rural population, and has improved the use of healthcare by insured rural residents [28,29]. However, reimbursement is low, and ambulatory patients with chronic diseases have limited financial protection, and still have to pay considerable amounts out of pocket [30-32].

It seems inevitable that this will impact on completion rates. Using surveys, there is a large variation in estimates of TB completion. The national TB survey in 2000 reported a low adherence rate, particularly low in the general health system. In recent studies reported here, the results are the proportion of patients not taking drugs at the time of the survey: the cumulative failure to complete is likely to be higher. Hence, the adherence on average was not so optimistic. What these data do is generate a degree of uncertainty around true completion rates.

TB treatment completion rate from the independent studies reported in this review contrast with published health service statistics which show very good performance [33]. The Ministry of Health in China and others have very clear targets for detection and treatment, set by government and endorsed by World Health Organization. It may be that the actual health information systems do not operate as well as expected, and where there are gaps in the data, health workers worldwide tend to take the most optimistic standpoint in their estimates. Thus the imputed completion rates may be overly optimistic. This we have found in a study in Chongqing [19], although there has been very little systematic assessment of the extent of this problem.

Given the number of patients in China, poor or irregular adherence could have massive public health implications, potentially increasing the risk of multi-drug resistant TB and extremely drug-resistant TB developing. This will cause a worsening of the TB epidemic in areas of China, and will increase the risk of morbidity and mortality among the poor as well as the costs associated with illness.

\section{Conclusions}

Despite a policy of free drug treatment for TB in China, health services charge all income groups, and costs are high. Adherence measured in cross sectional surveys is often low, and the cumulative failure to adhere is likely to be much higher. It is important that there is an open debate between health providers, the Ministry of Health, the World Health Organization and donors about these realities, and discussion about the public health impact. Policy makers need to consider appropriate steps to document further problems with adherence, charging, and how best to improve TB treatment delivery in poor patients.

\section{Acknowledgements}

This research was funded by the UK Department for International Development for the benefit of the poor in developing countries.

\section{Author details}

${ }^{1}$ School of Public Health, Chongqing Medical University, Chongqing, China. ${ }^{2}$ Department of Public Health, University of Helsinki, Helsinki, Finland.

${ }^{3}$ Liverpool School of Tropical Medicine, Liverpool, UK. ${ }^{4}$ School of Public Health, Peking University, Beijing, China. ${ }^{5}$ World Health Organization, Geneva, Switzerland. 


\section{Authors' contributions}

This review was designed collectively. QL retrieved references, applied inclusion criteria and extracted data. PG and HS assessed the eligibility of the retrieved papers, participated in the data analysis, results interpretation and the review writing. ST and TZ advised on data presentation interpretation, and contributed to the manuscript. All authors read and approved the final manuscript.

\section{Competing interests}

The authors declare that they have no competing interests.

Received: 2 December 2010 Accepted: 26 May 2011

Published: 26 May 2011

\section{References}

1. Tang S, Squire SB: What lessons can be drawn from tuberculosis (TB) Control in China in the 1990s? An analysis from a health system perspective. Health Policy 2005, 72(1):93-104.

2. Zignol M, Hosseini MS, Wright $A$, Weezenbeek $C L$, Nunn $P$, Watt $C J$ Williams BG, Dye C: Global Incidence of Multidrug-Resistant Tuberculosis. The Journal of Infectious Diseases 2006, 194(4):479-8.

3. Yip W, Hsiao WC: The Chinese health system at a crossroads. Health Affairs 2008, 27(2):460-468.

4. Bogg L, Dong HJ, Wang KL, Cai WW, Diwan V: The Cost of Coverage: Rural Health Insurance in China. Health Policy and Planning 1996, 11(3):238-252.

5. Wang L, Liu J, Chin DP: Progress in tuberculosis control and the evolving public-health system in China. Lancet 2007, 369:691-696.

6. Xu B: Access to tuberculosis care in rural China-comparing the impact of alternative control projects. PhD thesis Stockholm: Karolinska institute; 2006, 13-15.

7. Murray CJL: Results of directly observed short-course chemotherapy in 112842 Chinese patients with smear-positive tuberculosis. Lancet 1996, 347(8998):358-362

8. Liu Q, Smith H, Wang Y, Tang S, Wang Q, Garner P: Tuberculosis patient expenditure on drugs and tests in subsidised, public services in China: a descriptive study. Tropical Medicine and International Health 2010, 15(1):26-32.

9. Zhan S, Wang L, Yin A, Blas E: Revenue-driven in TB control-three cases in China. International Journal of Health Planning and Management 2004, 19(Suppl 1):S63-78.

10. Meng Q, Li R, Cheng G, Blas E: Provision and financial burden of TB services in a financially decentralized system: a case study from Shandong, China. International Journal of Health Planning and Management 2004, 19(Suppl 1):S45-62

11. Jackson S, Sleigh AC, Wang GJ, Liu XL: Poverty and the economic effects of TB in rural China. International Journal of Tuberculosis and Lung Disease 2006, 10(10):1104-10.

12. Zhang T, Tang S, Gao J, Whitehead M: Persistent problems of access to appropriate, affordable TB services in rural China: experiences of different socio-economic groups. BMC Public Health 2007, 7:19.

13. Xu L, Gai R, Wang X, Liu Z, Cheng J, Zhou C, Liu J, Zhang H, Li H, Li H, Tang W: Socio-economic factors affecting the success of tuberculosis treatment in six counties of Shandong Province, China. International Journal of Tuberculosis and Lung Disease 2010, 14(4):440-446.

14. Liu X, Thomson R, Gong Y, Zhao F, Squire B, Tolhurst R, Zhao X, Yan F, Tang S: How affordable are tuberculosis diagnosis and treatment in rural China? An analysis from community and tuberculosis patient perspectives. Tropical Medicine and International Health 2007, 12(12):1464-1471.

15. Xu B, Dong HJ, Zhao Q, Bogg L: DOTS in China-removing barriers or moving barriers? Health Policy Plan 2006, 21(5):365-372.

16. Huang $F$, Wang $L$, Yang $H$, Bai $L$ : Analysis of cost for TB patients in 10 counties, Hunan. Chinese Journal of Antituberculosis 2009, 31(8):449-453, (in Chinese).

17. National Technical Steering Group of the Epidemiological Sampling Survey: Report of Fourth National Epidemiological Sampling Survey of Tuberculosis in 2000. Chinese Journal of Tuberculosis and Respiratory Diseases 2002, 25(1):3-7, (in Chinese).

18. Wang $Y Y$, Liabsuetrakul $T$, Chongsuvivatwong $V$, Pungrassami $P, H e C Y$, Ren $L$, Yang HZ: Under- and over-consumption intermittent TB treatment among rural TB patients in south-west China. International Journal of Tuberculosis and Lung Disease 2007, 11(12):1345-1351.

19. Hu D, Liu X, Chen J, Wang Y, Wang T, Zeng W, Smith H, Garner P: Direct observation and adherence to tuberculosis treatment in Chongqing, China: a descriptive study. Health Policy and Planning 2008, 23(1):43-55.

20. Xu W, Lu W, Zhou Y, Zhu L, Shen H, Wang J: Adherence to antituberculosis treatment among pulmonary tuberculosis patients: a qualitative and quantitative study. BMC Health Services Research 2009, 9:169.

21. Wang J, Shen $\mathrm{H}$ : Direct observation and completion of treatment of tuberculosis in rural areas of China. Scandinavian Journal of Public Health 2009, 37:304-309.

22. Ai X, Men $K$, Guo L, Zhang $T$, Zhao $Y$, Sun $X$, Zhang $H$, He G, van der Werf MJ, van den Hof S: Factors associated with low cure rate of tuberculosis in remote poor areas of Shaanxi Province, China: a case control study. BMC Public Health 2010, 10:112

23. Sun B, Wang WB, Jiao JH, Zhu FD, Dan YM, Dong Q, Xu B, Pan ZB, Yan CH: Research of TB treatment compliance and socio-economic and clinical factors influencing it in Guanyun County. Chinese Primary Health Care 2007, 21(1):58-60, (in Chinese).

24. Xu B, Fochsen G, Xiu Y, Thorson A, Kemp JR, Jiang QW: Perceptions and experiences of health care seeking and access to TB care-a qualitative study in rural Jiangsu province, China. Health Policy 2004, 69(2):139-149.

25. Wei X, Liang X, Liu F, Walley J, Dong B: Decentralising tuberculosis services from county tuberculosis dispensaries to township hospitals in China: an intervention study. International Journal of Tuberculosis and Lung Disease 2008, 12(5):538-547.

26. Yao H, Wei X, Liu J, Zhao J, Hu D, Walley JD: Evaluation the effects of providing financial incentives to tuberculosis patients and health providers in China. International Journal of Tuberculosis and Lung Disease 2008, 12(10):1166-1172

27. Wei X, Chen J, Chen P, Newell J, Li H, Sun C, Mei J, Walley JD: Barriers to TB care for rural-to-urban migrant TB patients in Shanghai: a qualitative study. Tropical Medicine and International Health 2009, 14(7):754-760.

28. Wagstaff A, Lindelow M, Gao J, Xu L, Qian J: Extending health insurance to the rural population: An impact evaluation of China's new cooperative medical scheme. Journal of Health Economics 2009, 28:1-19.

29. Yu B, Meng Q, Collins C, Tolhurst R, Tang S, Yan F, Bogg L, Liu X: How does the New Cooperative Scheme influence health service utilization? A study in two provinces in rural China. BMC Health Services Research 2010, 10:116.

30. Sun Q, Liu X, Meng Q, Tang S, Yu B, Tolhurst R: Evaluating the financial protection of patients with chronic disease by health insurance in rural China. International Journal for Equity in Health 2009, 8:42.

31. Wagstaff A, Lindelow M: Can insurance increase financial risk? The curious case of health insurance in China. Journal of Health Economics 2008, 27(4):990-1005

32. Winnie $Y$, Hsiao W, Non-evidence-based policy: How effective is China's new cooperative medical scheme in reducing medical impoverishment? Social Science \& Medicine 2009, 68:201-209.

33. World Health Organization: Global Tuberculosis Control Epidemiology, Strategy, Financing: WHO report 2009. Geneva: World Health Organisation; 2009

\section{Pre-publication history}

The pre-publication history for this paper can be accessed here: http://www.biomedcentral.com/1471-2458/11/393/prepub

doi:10.1186/1471-2458-11-393

Cite this article as: Long et al:: Patient medical costs for tuberculosis treatment and impact on adherence in China: a systematic review. BMC Public Health 2011 11:393. 